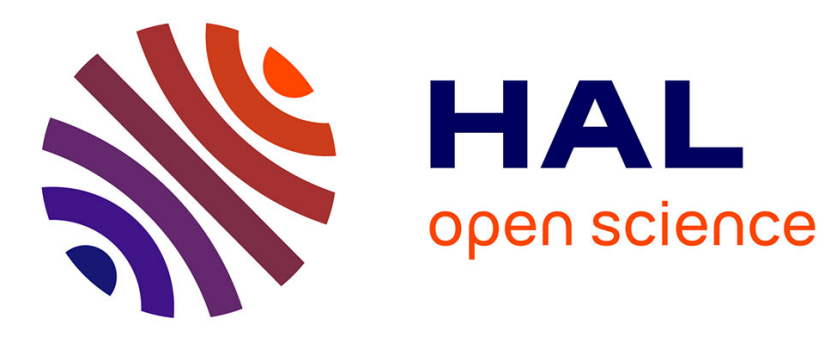

\title{
Les félicités du capital en santé
}

\author{
Catherine Bourgain, Maurice Cassier, Jean-Paul Gaudillière, Pierre-André \\ Juven
}

\section{To cite this version:}

Catherine Bourgain, Maurice Cassier, Jean-Paul Gaudillière, Pierre-André Juven. Les félicités du capital en santé: Épanouissement, épreuves et tensions critiques des industries pharmaceutiques aux Nords et aux Suds. Revue Française de Socio-Economie, 2021, La santé, miroir des sociétés, 26, pp.127-147. 10.3917/rfse.026.0127 . halshs-03464016

\section{HAL Id: halshs-03464016 https://shs.hal.science/halshs-03464016}

Submitted on 2 Dec 2021

HAL is a multi-disciplinary open access archive for the deposit and dissemination of scientific research documents, whether they are published or not. The documents may come from teaching and research institutions in France or abroad, or from public or private research centers.
L'archive ouverte pluridisciplinaire HAL, est destinée au dépôt et à la diffusion de documents scientifiques de niveau recherche, publiés ou non, émanant des établissements d'enseignement et de recherche français ou étrangers, des laboratoires publics ou privés. 


\title{
$\underline{\text { Revue Francaise de Socio-Économie, 2021/1 (n²6), pages } 127 \text { à }}$
}

$\underline{147}$

\author{
Les félicités du capital en santé \\ Épanouissement, épreuves et tensions critiques des industries pharmaceutiques aux \\ Nords et aux Suds
}

Catherine Bourgain (CERMES3 - INSERM)

Maurice Cassier (CERMES3 - CNRS)

Jean-Paul Gaudillière (CERMES3- INSERM)

Pierre-André Juven (CERMES3- CNRS)

\section{Résumé :}

Alors que le terme de « crise » est sans cesse mobilisé depuis le milieu des années 2000 pour décrire une industrie pharmaceutique financiarisée et spéculant sur un nombre limité d'innovations à valeur ajoutée clinique importante, cet article propose d'élargir la perspective en considérant les capitalismes pharmaceutiques des Nords et des Suds dans leur diversité. Nous présentons une analyse socio-historique de plusieurs modes de déploiements de ces industries de santé et des conditions de "félicité » de leur modèle scientifique et financier. Insistant sur l'importance des infrastructures politiques de construction des marchés et sur les ordres institutionnels dans lesquels les industries s'insèrent (et qu'elles contribuent à façonner), nous montrons qu'il est possible de lire le développement des capitalismes pharmaceutiques à l'aune de trois critères : le degré de financiarisation, les formes de propriété intellectuelle et la place prise par les biotechnologies. Cette analyse remet en cause la division entre des Nords industriels et innovateurs d'une part et des Suds gérant la pénurie d'autre part.

Mots-clefs : Industrie pharmaceutique ; financiarisation ; brevet ; savoirs traditionnels ; crise ; régulation; Nord(s); $\operatorname{Sud}(\mathrm{s})$.

\section{Title}

The felicities of health capital

Growth, trials and tensions in the pharmaceutical industries of the Global North and South

\begin{abstract}
While the term "crisis" has constantly been used since the mid-2000s to describe a financialized pharmaceutical industry speculating on a limited number of innovations of high clinical added value, this article broadens the perspective by considering the pharmaceutical capitalism of the Global North and South in all its diversity. We present a socio-historical analysis of several modes of implementation of these health industries and the felicity conditions of their scientific and financial model. While stressing the importance of the political infrastructures underpinning the construction of markets, and the institutional orders within which the industries exist (and which they help to shape), we show that it is possible to analyse the development of pharmaceutical capitalism in terms of three criteria: the degree of
\end{abstract}


financialization, the forms of intellectual property, and the role of biotechnologies. This analysis calls into question the division between the industrial and innovative North, on the one hand, and the South that has to manage shortages, on the other.

Key words : Pharmaceutical industry; financialisation; patent; traditional knowledge; crisis; regulation; North(s); South(s).

\section{Introduction}

En juillet 2017, le New-York Times publie un article sur une structure au modèle économique relativement nouveau dans le monde de la santé, Royalty Pharma, "The Private Equity Firm That Quietly Profits on Top-Selling Drugs » ${ }^{1}$. Cette " société d'investissement privé qui spécule tranquillement sur les médicaments les plus vendus » a été fondée en 1996. «Notre stratégie est simple: nous achetons des droits de redevance sur les marchés pharmaceutiques et des biotechnologies afin d'ajouter les intérêts de ces investissements comme partie intégrante de votre portefeuille d'actions $»^{2}$. En 2014, Royalty Pharma a déboursé plus de trois milliards de dollars pour disposer, entre autres, des droits du Kalydeco, un médicament dans le traitement de la mucoviscidose commercialisé par le laboratoire pharmaceutique Vertex. Pour de nombreux analystes, ces évènements sont la marque de l'émergence d'une bulle spéculative similaire à celle que les marchés ont connu au début des années 2000 avec l'internet. Ainsi, « l'Américain Gilead Sciences, qui compte 4000 salariés, produit deux traitements onéreux contre l'hépatite C (le Sovaldi et l'Harvoni), et vaut près de 150 milliards de dollars en Bourse, pour un chiffre d'affaires de 11 Mds de dollars. Ce sont les mêmes ordres de grandeur que le géant français Sanofi (131 milliards de capitalisation et 30 milliards de chiffre d'affaires en 2017), propriétaire notamment du doliprane, et ses 110000 salariés $»^{3}$.

Les travaux conduits en économie et en sociologie s'accordent à relever une situation critique des États-providence au Nord dont une manifestation est la difficulté croissante à financer l'accès à certaines innovations thérapeutiques (Brissaud et Juven, 2020). Mis en place dans les Nords dans l'après-guerre avec l'industrialisation massive du secteur, la structure dominante des systèmes de santé présente trois caractéristiques majeures : l'hégémonie de la biomédecine et la domination d'un modèle d'innovation thérapeutique centré sur le criblage ("screening ») des molécules; le contrôle de la recherche et du développement des médicaments par les entreprises et la consolidation de leur appropriation par la propriété intellectuelle ; la socialisation des coûts du médicament par les systèmes d'assurances maladie et donc la création d'un marché garanti. À partir de la fin des années 1970, ce modèle a été profondément affecté par les restructurations de l'industrie (fusions et externalisations), l'essor des biotechnologies comme nouvelle frontière de recherche et de valorisation, le renforcement du système des brevets et la financiarisation du secteur. Dans les Suds en revanche, des capitalismes alternatifs émergent à la même période. L'intégration des médecines traditionnelles devient un enjeu de santé publique dans certains pays d'Asie et un autre type d'encastrement structurel et marchand des pouvoirs publics et de l'industrie de santé se fait jour. Un capitalisme de la copie s'impose dans des pays en Inde ou au Brésil.

\footnotetext{
${ }^{1}$ Randall Smith, « The Private Equity Firm That Quietly Profits on Top-Selling Drugs », New-York Times, 8 juillet 2017. En ligne : https://www.nytimes.com/2017/07/08/business/dealbook/drug-prices-private-equity.html

${ }^{2}$ https://www.royaltypharma.com/our-approach

${ }^{3}$ « Risque de bulle spéculative autour des biotechnologies », L’Humanité, 5 avril 2015. En fait, Si le CA de Gilead était bien de 11 mds en 2013, il a triplé en 2015-2016 avec la mise sur le marché du Sovaldi et d'Harvoni.
} 
En première approximation, il semble que les capitalismes pharmaceutiques s'accommodent plutôt bien des structures économiques et politiques des Nords et s'adaptent moins bien à celles des Suds et à leurs difficultés de financement de la santé. En dehors d'épisodes exceptionnels comme celui du VIH (Dalgalarrondo, 2004), au Nord, le marché assumerait son rôle de promoteur d'innovations et les systèmes collectivisés des dépenses de santé mis en place après 1945 garantiraient la mise sur le marché et la prise en charge de traitements innovants. A contrario, les pays du Sud seraient soumis en permanence à des " rationnements », ne bénéficieraient que dans un second temps des innovations développées au Nord et ne disposeraient que de formes très imparfaites de protection collective avec un système public centré sur les besoins tenus pour « prioritaires ». Notre article remet en cause cette vision en soulignant que l'image des Nords épanouis et des Suds éprouvés est d'autant moins d'actualité que : 1) le modèle de capitalisme pharmaceutique des pays du Nord est soumis à de fortes contraintes du fait même des orientations qui le caractérisent depuis les années 1970 ;2) des formes originales de capitalisme pharmaceutique ont surgi au Sud, entrant en concurrence avec la grande industrie du Nord et tendant à en accentuer les tensions si ce n'est la « crise ».

Dans l'ouvrage de référence qu'il consacre à la théorie de la régulation, Robert Boyer note, que les crises partagent un « petit nombre de mécanismes de base qui, à un certain niveau d'abstraction, sont dotées d'invariances" (Boyer, 2015, p. 80). Deux points sont particulièrement relevés. Premièrement, «la croissance n'est pas le résultat garanti de l'application du progrès technique, mais l'expression de la cohérence d'un ensemble de formes institutionnelles $\gg($ p. 81). Les conditions de félicité du capitalisme pharmaceutique ne dépendent pas seulement de la capacité à produire des innovations mais également de la capacité à structurer socialement et politiquement un marché qui fasse de la production un objet rentable et à l'abri de concurrences fragilisant l'ordre ainsi établi. Deuxièmement, les crises en régime capitalistique ne sauraient être analysées uniquement en termes d' "imperfections de marchés » et d' «erreurs de politique économique ». Prolongeant une partie des analyses marxistes, la théorie de la régulation soutient au contraire que les crises sont « l'expression de tendances d'un mode de régulation et d'un régime d'accumulation» (p. 81). Dans cet article, nous montrons que les modes de régulation et le régime d'accumulation de l'industrie pharmaceutique dans les Nords s'ils permettent un certain «dynamisme " (l'existence même de Royalty Pharma en atteste), éprouvent également les systèmes de santé.

L'analyse croisée Nords/Suds que nous proposons montre ainsi que les conditions de félicité du capital dans le domaine pharmaceutique ne sont pas univoques. Le cas du capitalisme indien valorisant la médecine ayurvédique montre que le développement d'un capitalisme pharmaceutique peut se faire par l'industrialisation des savoirs traditionnels. A contrario, les évolutions récentes des marchés du médicament interrogent le modèle d'innovation des Nords, centré sur l'invention de nouvelles molécules adossées aux biotechnologies, et leur contrôle par le monopole de propriété intellectuelle. Pour analyser les agencements marchands (Callon, 2017) conduisant à l'épanouissement et à la mise à l'épreuve de différents capitalismes pharmaceutiques, nous proposons dans cet article de raisonner autour de cas emblématiques qui portent aussi bien sur des entreprises (Gilead, Genentech, Cipla, Himalaya) que sur des produits (Sovaldi, Triomune, Menosan, Truvada).

\section{Science, brevet et finance : le nouveau régime du capitalisme biopharmaceutique depuis les années 1970}


Le secteur pharmaceutique connaît à partir des années 1970 un double bouleversement résultant des transformations du régime juridique des brevets. Le premier bouleversement a trait à la révolution technologique que connaît le secteur qui passe d'un modèle d'innovation et de production reposant sur des technologies chimiques à un modèle basé sur les biotechnologies. Le second bouleversement porte sur les modes de financement et sur la financiarisation de l'industrie (Abecassis et Coutinet, 2018). Cette financiarisation trouve dans le fort développement du capital-risque la marque de sa désintégration et de son encastrement toujours plus poussé aux marchés financiers. Genentech et Gilead que nous prenons ici comme cas d'étude incarnent ces transformations. Ces deux firmes sont deux faces d'une même médaille. Toutes deux sont marquées par le choix stratégique des biotechnologies et par une financiarisation des modes de financement. Mais la première est une structure basée sur le capital-risque et la seconde mise sur des stratégies d'acquisition et de sous-traitance. Genentech avait opté pour une forte intégration de la $R \& D$ et de l'industrie, tandis que Gilead poursuit un modèle de $R \& D$ et de production marquée par une forte externalisation.

\section{La recherche publique et les assurances sociales dans l'expansion du capitalisme au nord}

L'appropriation de la science et des innovations biomédicales est centrale pour les industries de santé. L'industrie pharmaceutique a constitué dès le début du $20^{\text {ème }}$ siècle ses propres laboratoires de recherche et développement (R\&D) (Liebenau, 1987 ; Swann, 1988 ; Quirke, 2008). La croissance simultanée des départements de propriété intellectuelle témoigne de la centralité des enjeux de contrôle des innovations produites. De fait, après 1945, la montée en puissance des investissements de R\&D, internes et externes, des firmes pharmaceutiques s'appuient sur le déploiement d'un modèle d'innovation « verticalisé », focalisé sur le screening à grande échelle des molécules (Gaudillière, 2015), et sur la généralisation des brevets de produits.

La croissance des profits pharmaceutiques et de l'innovation n'a pas été que le résultat d'investissements internes. Elle a aussi bénéficié de la mise en place des assurances sociales à partir des années 1940 (Chauveau, 1999) ainsi que de la croissance vertigineuse des dépenses publiques de recherche dans le champ de la santé depuis les années 1950, devenues principales dépenses non militaires des États-Unis dans les années $2000^{4}$. L'impact des recherches conduites par les National Institutes of Health (NIH), la principale institution publique de soutien à la recherche biomédicale, sur l'industrie pharmaceutique ${ }^{5}$ conforte l'idée que ce domaine d'activité capitalistique prend appui sur des ressources publiques et sur un «ordre institutionnel » favorable (Jullien, Smith, 2012). La captation des recherches académiques se fait pour un coût très modique, tandis que les firmes supportent les coûts d'absorption des savoirs et de développement industriel et clinique. Les biographies du Taxol (Walsh et Goodman, 2001) et de l'AZT illustrent l'appropriation exclusive de travaux publics par des laboratoires privés, en dépit de contestations émanant du Congrès des États-Unis et des NIH (Salbu, 1993). Si le financement public de la recherche biomédicale est plus limité en France, dans les années 60 à 80, les recherches conduites par le CNRS sur la chimie des substances naturelles ont, par exemple, été essentielles au développement de nouveaux traitements dans le champ du cancer par Rhône Poulenc et Pierre Fabre (Walsh et Leroux, 2014).

\footnotetext{
${ }^{4}$ Historical Trends in Federal R\&D, American Association for the Advancement of Science, 2018.

${ }^{5}$ « Of the 21 most important drugs introduced between 1965 and 1992, 15 were developed using knowledge and techniques from federally funded research ", The benefits of Medical Research and the role of NIH, US Senate, April 2000, 64 p.
} 
Les systèmes de dépenses de santé collectivisées constituent un double point d'appui pour les industries de santé : (1) les transferts et les retombées des dépenses publiques de recherche dans le domaine de la santé augmentent la productivité de l'innovation privée et (2) la mutualisation des paiements limite l'impact des prix de monopole sur l'accès aux thérapies. Cette double opération n'est cependant pas sans limite. Dès la fin des années 1970, elle s'accompagne d'une croissance des dépenses réglementaires de mise sur le marché et d'une baisse de la productivité des dépenses de R\&D des firmes. On peut documenter l'émergence, en réponse, d'un nouveau régime d'innovation et de capitalisation de la science à partir du début des années 1980, qui va s'imposer progressivement sans pour autant éclipser entièrement le régime précédent. Ce régime capitalistique et scientifique se développe d'abord aux États-Unis autour des grandes universités et des NIH.

L'un des cas les plus emblématiques de ce modèle est la création de la firme Genentech en 1976 à partir de recherches pionnières de l'Université de Stanford sur l'ingénierie génétique. Les récits qu'en font ses acteurs, le chercheur Herbert Boyer et des fondateurs du capital risque Thomas Perkins et Robert Swanson, sont particulièrement éclairants ${ }^{6}$. La création de capital par Genentech est considérable : estimée à 300 millions de dollars en 1980, l'entreprise est acquise par Roche en 2009 pour 47 milliards de dollars. Les premiers développements portent sur des technologies de production d'insuline recombinante. Ils se font à l'Université et sont financés par un partenariat avec Eli Lilly, firme implantée sur ce marché. Les fonds apportés par le « capital risqueur » sont au départ très modestes, autour de $\$ 200000$. Quatre ans plus tard, quand Genentech est mise sur le marché public, la valeur des premières actions a déjà été multipliée par 800. La société de R\&D intègre graduellement l'industrie pharmaceutique. Elle est emblématique des product companies (Cockburn, 2003) qui se développent à la même période, en mettant sur le marché des médicaments ou des tests génétiques (Qiagen, Myriad Genetics, Genzyme).

\section{Le capital risque et l'intangibilité à l'appui des biotechnologies}

La croissance du secteur autonome des biotechnologies, entre champ académique et firmes pharmaceutiques, se fait en interaction avec le secteur du capital risque, en plein développement. L'originalité de ce dernier tient à ce qu'il intervient directement dans le capital de la firme et y détient un pouvoir de gouvernement : Robert Swanson sera ainsi le chairman du Board de Genentech pendant plus de vingt ans. Cette rencontre entre innovations en santé et dispositifs de marchés financiers ne se fait pas in abstracto. Elle s'insère dans un cadre réglementaire indispensable à son épanouissement. Les fonds du capital risque bénéficient directement de la décision du Département d'État du Travail en 1979 (Lerner, 1994) permettant leur abondement par les fonds de pension. Autre réforme juridique décisive, celle autorisant, en 1984, des sociétés de nouvelles technologies n'ayant pas généré de revenus nets d'entrer en bourse (aussi appelée « l'option 2 du Nasdaq ») ${ }^{7}$. Ce fut précisément le cas de Genentech ${ }^{8}$.

Ces projets sont rendus possible par une « valorisation du capital intangible » constitué en premier lieu par les droits de propriété intellectuelle des firmes. L'économiste financier, Elli

\footnotetext{
${ }^{6}$ Oral Histories on Biotechnology, Program in the History of the Biological Sciences and Biotechnology, University of California Berkeley.

${ }^{7}$ National Association of Securities Dealers: Notice to members 84-26, 11 may 1984: « The second alternative proposed for NMS inclusion is for companies without a net income ».

${ }^{8}$ Thomas J. Perkins, "Kleiner Perkins, Venture Capital, and the Chairmanship of Genentech, 1976-1995," an oral history conducted in 2001 by Glenn E. Bugos for the Regional Oral History Office, The Bancroft Library, University of California, Berkeley, 2002.
} 
Malki (Malki, 1997) montre que la valeur des sociétés de biotechnologie est un problème déroutant dès lors que leurs revenus sont négatifs et qu'elles s'apprécient très positivement sur les marchés financiers. Il en conclut qu'elles se financent sur la base des promesses de revenus futurs autorisées par leurs actifs de propriété intellectuelle et propose une méthode d'évaluation de ces actifs intangibles. Ce sont en conséquence le plus souvent les cabinets de conseil en propriété intellectuelle qui rédigent les dossiers d'introduction des sociétés de biotechnologie sur les marchés financiers. Le régime des promesses technoscientifiques (Joly, 2010) n'est donc pas contemporain mais bien inscrit dans le développement des biotechnologies par les marchés financiers et ce, dès les années 1970.

La construction de ce capital intangible est associée à l'extension des normes de brevetabilité à une foule d'entités du vivant plus ou moins modifiées et protégées par des brevets de produit dont le pouvoir de monopole est très étendu (Cassier et Gaudillière, 2000 ; Coriat et Orsi, 2002). Ainsi, les brevets princeps de Boyer et Cohen à l'Université de Stanford portent sur des « chimères moléculaires biologiquement actives » pour produire des protéines médicaments. David Mowery montre que le nombre des brevets revendiqués par les universités croit dès les années 1970, en relation avec l'essor des biotechnologies, et que la tendance se renforce avec le Bayh Dole Act de 1980 (Mowery et Ziedonis, 2000). La nouveauté de ce système propriétaire tient encore à la globalisation de la rente qu'il permet, avec la convergence des critères de brevetabilité des grands offices de brevets (US Patent and Trademark Office, European Patent Office, offices des brevets du Japon), dès les années 1980, et l'adoption des accords sur les Aspects des droits de propriété intellectuelle (ADPIC) piliers de l'Organisation Mondiale du Commerce (OMC) en 1994. L'émergence d'une rente globale soulignée par Philippe Aghion ${ }^{9}$ est revendiquée par le capitalisme pharmaceutique.

Le développement du capital-risque constitue donc l'une des modalités privilégiées de conversion des firmes pharmaceutiques aux logiques de financiarisation. La systématisation des fusions-acquisitions et la désintégration de l'organisation pharmaceutique, que nous discutons après, sont d'autres marqueurs forts de cette même tendance à l'encastrement d'un domaine industriel dans des mécanismes financiers. Si le déploiement du capital-risque a assuré la conversion de l'activité pharmaceutique au régime des promesses technoscientifiques, l'exigence associée d'une rentabilité élevée pour satisfaire les investisseurs, a produit une inflation du marché pharmaceutique et des tensions croissantes sur les prix. L'évolution des prix de l'insuline illustre ces évolutions. Ce médicament, dont le marché est l'un des plus étendus et plus rémunérateurs, fut découvert et breveté dans les années 1920. En 1978, Genentech dépose le brevet de son inuline recombinante et Eli Lilly la met sur le marché en 1982. Depuis cette époque, les prix de l'insuline devenue biotechnologique demeurent très élevés par l'effet de l'evergreening des brevets, d'une structure d'oligopole réduite à trois firmes (Eli Lilly, Novo Nordisk et Sanofi) et des difficultés d'émergence d'un marché des biosimilaires (Greene, Riggs, 2015).

\section{Désintégration pharmaceutique}

Alors que dans le modèle précédant le déploiement des biotechnologies, les firmes pharmaceutiques acquièrent auprès du secteur académique des outils de recherche et des nouvelles molécules pour un coût modique tout en poursuivant leur propre R\&D interne, un nouveau modèle d'organisation apparait au début des années 2000. Il peut être analysé comme

${ }^{9}$ Philippe Aghion, Le retour de la rente ? Economie des institutions, de l'innovation et de la croissance, Collège de France, 21 novembre 2017. 
une «désintégration verticale de l'industrie pharmaceutique » selon l'économiste Ian Cockburn. Faisant fond sur l'épuisement du modèle du screening et du contrôle direct de la R\&D (Gaudillière, 2021), cette désintégration suppose d'acquérir ces outils et nouvelles molécules auprès des sociétés de biotechnologie, financées par le capital financier, et soumises à des exigences de retours élevés sur investissement. Ce déplacement du modèle capitalistique des firmes va de pair avec une augmentation du coût en capital des innovations : leur développement suppose de multiples opérations de rachats et de valorisation sur les marchés (Cassier, 2021). Dès 2003, Ian Cokburn posait la question : "Que va-t-il arriver aux prix des produits qui incorporent directement ou indirectement ces inventions?» (Cokburn, 2003).

La trajectoire de Gilead Sciences éclaire ce nouveau capitalisme pharmaceutique qui réduit ses investissements de $R \& D$ en interne pour acquérir à l'extérieur des innovations thérapeutiques confirmées par des essais cliniques. Gilead Sciences, particulièrement visible depuis 2014 pour le prix excessivement élevé du Sovaldi, un médicament pour le traitement l'hépatite $\mathrm{C}$, et pour l'énorme volume de profits engrangés en un temps très court grâce à sa vente, appartient à une nouvelle génération de sociétés de R\&D. À l'instar des autres sociétés de biotechnologie, Gilead est demeurée durant quinze ans sans faire le moindre profit. Entre 1987 et 2002, elle était financée par le capital-risque et par le Nasdaq. La première molécule hautement rentable développée par Gilead, le tenofovir, une des molécules les plus utilisées pour traiter le VIH/sida, également active contre l'hépatite $\mathrm{B}$, est en fait une invention de la recherche académique tchèque et belge (Holy et De Clerq), licenciée de manière exclusive à Gilead en 1991 (Veras, 2014). L'appropriation du tenofovir est typique des réseaux académie/industrie pharmaceutique décrits plus haut.

La société californienne utilise en revanche un autre modèle d'appropriation pour organiser sa croissance à partir des années 2000, et incarne le modèle de l'industrie pharmaceutique « désintégrée » de Cockburn. Entre 2003 et 2001, Gilead qui sous-traitait déjà la quasi-totalité de sa production n'achète pas moins de huit sociétés, dont Pharmasset le développeur du sofosbuvir qui deviendra le Sovaldi. L’enquête effectuée par le Sénat des ÉtatsUnis sur la formation du prix du Sovaldi éclaire le coût en capital de ce rachat. Elle se fonde notamment sur les matrices utilisées par Gilead pour associer hypothèses de prix des actions et hypothèses de prix de la molécule, brusquement réévaluées par la publication d'essais cliniques favorables. Le prix d'acquisition de Pharmasset - 11 Mds de dollars- est ainsi totalement déconnecté du coût en capital de l'innovation, estimé à 356 millions de dollars (JAMA, August 13, 2014). En conséquence, le prix du Sovaldi est indexé sur la capitalisation de Pharmasset, rachetée par Gilead, et aux normes de profitabilité, à maximiser. Cette enquête révèle que la formation du prix s'est basée sur une évaluation des prix maximum que les médecins et les payeurs étaient disposés à consentir ${ }^{10}$ : le prix fixé aux États-Unis sera de $\$ 84000$ le traitement.

Le cas de Gilead éclaire les évolutions d'une partie de l'industrie pharmaceutique, en matière de financiarisation des activités et de restructuration organisationnelle de la chaîne productive. Le passage d'une économie de l'innovation chimique à une économie de l'innovation biomoléculaire a ainsi permis de profonds bouleversements dans les modalités de fonctionnement de la recherche, du développement et de la production.

\section{L'envers de la propriété : les capitalismes de la copie aux Suds}

\footnotetext{
${ }^{10}$ Orrin G. Hatch, Ron Wyden, The Price of Sovaldi and its Impact on the U.S. Health Care System, 2015.
} 
Dans les années 1970, un autre modèle pharmaceutique se développe dans les pays émergents, en Inde, en Chine, au Brésil ou en Argentine. Les nouveaux champions de ce capitalisme de la copie sont Cipla, Ranbaxy ou Dr Reddy et ils ont aujourd'hui acquis une dimension internationale. Tandis que l'accumulation du capital biopharmaceutique dans les Nords suppose et bénéficie de l'extension des droits de propriété intellectuelle, le capitalisme de la copie bénéficie au contraire de leur absence (en Chine jusque dans les années 1990) ou de leur affaiblissement.

\section{Construction d'un capitalisme de la copie : le cas de l'Inde}

En 1970 et 1971, l'Inde et le Brésil adoptent des lois qui placent les médicaments en dehors de la brevetabilité, ouvrant ainsi un espace pour la copie des inventions étrangères. Les économistes ont mesuré la croissance vertigineuse de l'industrie des génériques en Inde qui a découlé de cette exclusion des brevets de produits et de la limitation drastique des brevets de procédés jusqu'en 2005, quand l'Inde s'est conformée aux accords ADPIC de l'OMC (Chauduhri, 2005 ; Lanjouwe, 1997). Entre temps, le pays est devenu la troisième industrie pharmaceutique mondiale en volume, et la neuvième en valeur. Cette valeur a été multipliée par 150 tandis que le nombre de firmes passait de 1600 à 9000. Alors qu'au Nord les modèles industriels se convertissaient aux logiques des marchés financiers, les industries pharmaceutiques dans les Suds, tout en s'inscrivant dans des logiques capitalistiques de marché, optaient pour un développement bien moins liés aux techniques financières.

La part de marché des industries nationales, qui avait baissé entre 1952 et 1970 (de 62 à $32 \%$ ) en raison de l'emprise grandissante des multinationales contrôlant le flux des innovations (antibiotiques, néo sulfamides, etc.), grimpe à $77 \%$ en 2004 . La copie de molécules sophistiquées via le «reverse engineering » produit de nouveaux savoirs au sein des firmes imitatrices (Cassier et Correa, 2009). Les chimistes indiens acquièrent une forte expertise, qui leur permet de réduire les temps de reverse engineering et de raccourcir les délais de diffusion des copies après la mise sur le marché des nouveaux médicaments aux États-Unis (Scherer et Watal, 2001). De cinq ans en moyenne avant 1985, le temps de la copie passe à deux ans dans les années 1990. Lorsqu'en 2005, Roche met en cause la capacité de la société indienne Cipla à dupliquer le Tamiflu, elle souligne sa capacité d'expertise dans la reproduction des antirétroviraux ${ }^{11}$. De fait, à l'automne 2005, Cipla met sur le marché un générique du Tamiflu.

Plus largement, les firmes indiennes mettent à profit leur droit de libre copie pour inventer des combinaisons de molécules difficiles à réaliser dans l'économie propriétaire dès lors que les droits sont dispersés entre plusieurs laboratoires. Le coût de maître dans cette lignée d'invention est la formulation de la Triomune par Cipla, la première combinaison à dose fixe contre le VIH/sida, mise sur le marché en 2001. L'impact de cette innovation est double. Elle est plébiscitée par les patients qui voient leurs traitements facilités et la Triomune est commercialisée pour $\$ 300$ par an quand les antirétroviraux (ARVs) propriétaires sont vendus à $\$ 10$ 000. Par ailleurs, si les compétences des laboratoires indiens sont parfois contestées par les laboratoires propriétaires, ces derniers recourent en fait massivement à ces derniers pour s'approvisionner en « Active Pharmaceutical Ingredients », à moindre coût (Chaudhuri, 2005).

Cette stratégie d'un capitalisme de la copie va être renforcée par la recherche d'une reconnaissance internationale. Les grandes firmes indiennes de génériques entreprennent d'obtenir des certifications internationales de leurs produits auprès de la FDA et du système de

\footnotetext{
${ }^{11}$ Yusuf K. Hamied, Global Forum for Health Research, 21 october 2005.
} 
pré-qualification de l'OMS, mis en place au début des années 2000 pour garantir la qualité des médicaments génériques pour le $\mathrm{VIH}$, la malaria et la tuberculose, y compris pour des molécules sous brevets. Le Directeur Général de Cipla exposait ainsi : cela "prouve que nous [Cipla] adhérons aux bonnes pratiques de fabrications sur un pied d'égalité avec d'autres compagnies. [...] Maintenant les multinationales ne pourront plus nous apostropher de cette manière : ce sont les Indiens, ils viennent du Tiers-Monde, la qualité doit être douteuse ». En 2010 les firmes indiennes représentent 68\% des 267 médicaments pré-qualifiés (Lantenois et Coriat, 2014). Cette pré-qualification leur permet de vendre sur le marché des donateurs globaux $^{12}$. En 2008, les fabricants indiens fournissent 90\% des ARVs pour les pays du sud.

La firme Cipla a même développé un véritable activisme thérapeutique, comme en 2001 lors du procès de Pretoria intenté par 39 multinationales contre l'importation d'ARVs génériques par le gouvernement de l'Afrique du Sud, mais également pour produire des génériques du Tamiflu de Roche ou du Glivec de Novartis. L'anthropologue Kaushik Sunder Rajan (2017) a fait le portrait de cette société née en 1935 et symbole de l'industrie nationale indienne. Dès la fin des années 1960, la firme a intégré la production des principes actifs et la formulation des médicaments. L'ouverture du droit de copie en 1970 a renforcé sa croissance et en 2016 elle exporte $56 \%$ de sa production dont $1 / 3$ aux États-Unis et 1/3 en Afrique. Elle a acquis 2 sociétés aux États-Unis et investit simultanément en Afrique. Spécialisée dans le reverse engineering, elle a augmenté ses investissements de R\&D de 1\% dans les années 1990 à $6 \%$ en 2016.

\section{Les adaptations du modèle}

Le changement du régime de propriété intellectuelle en Inde en 2005, avec la reconnaissance des brevets de produits, a certes favorisé le retour des multinationales des Nords mais il a aussi généré une forte résistance pour défendre des espaces de copie. L’Office des brevets indiens s'est saisi des critères de brevetabilité restrictifs de la loi indienne pour faire tomber des brevets dans le domaine public et des firmes nationales ainsi que des associations de patients ont intensément utilisé le droit d'opposition pour faire invalider des brevets. Un des moments forts de cette résistance a été la décision de la Cour Suprême Indienne en avril 2013 de confirmer l'invalidation du brevet de Novartis sur le Glivec, justifiée par l'application des critères propres de brevetabilité de la République indienne et par la défense de l'intérêt de la santé publique ${ }^{13}$.

Les multinationales se sont efforcées d'apporter des réponses stratégiques à cette résistance indienne. En septembre 2014, Gilead Sciences décide de proposer des licences de fabrication de génériques du très controversé Sovaldi à sept laboratoires indiens, afin de contourner les oppositions juridiques initiées pour faire tomber son brevet. Ce réseau de licences volontaires permettait aussi de prévenir le recours à une licence obligatoire par le gouvernant indien. Le pays doit aussi faire face au défi du prix des médicaments et de l'accessibilité de ses génériques pour sa population pauvre. En 2011, un rapport sur la Couverture santé Universelle ${ }^{14}$ recommandait d'instaurer un contrôle des prix, d'étendre la liste des médicaments essentiels et d'utiliser les clauses de sauvegarde des accords internationaux sur la propriété intellectuelle pour pouvoir produire ces médicaments. Le paradoxe est que la

\footnotetext{
12 «Le marché des donateurs globaux » est une expression consacrée qui désigne le Fonds Mondial et toutes les fondations qui financent les achats de médicaments grâce à des subventions et donations (Clinton, Unitaid, etc).

${ }^{13}$ Novartis vs Union of India, Supreme Court of India, April 2013, 112 pages.

${ }^{14}$ High Level Expert Group Report On Universal Health Coverage for India, 2011.
} 
« pharmacie du monde », qui satisfait les besoins du marché mondial des génériques, n'est pas accessible pour une large part de sa population.

Les capitalismes nés de la copie en Inde et en Chine ont au fil du temps renforcé leurs capacités d'innovation, même si leur part dans le flux des inventions demeure limité. Les grands laboratoires de génériques ont augmenté leurs dépenses de R\&D. Ensuite, ces pays ont mobilisé leurs pharmacopées traditionnelles, soit pour reformuler et industrialiser des médicaments « traditionnels » jusque sur les marchés mondiaux (Pordié et Gaudillière, 2014), soit pour extraire les principes actifs des plantes en associant les laboratoires de chimie et les instituts de médecine traditionnelle. Il faut signaler la croissance accélérée des dépôts de brevets chinois depuis les années 2000, l'augmentation des investissements publics de R\&D ainsi que l'arrivée de sociétés de biotechnologie chinoises dans les investissements les plus importants de venture capital enregistrés en $2016^{15}$.

\section{L'intégration capitalistique des savoirs traditionnels}

Le capitalisme pharmaceutique indien ne se cantonne pas à la production de génériques. Depuis le tournant des années 2000, une autre forme de mondialisation et d'industrialisation des marchés de la santé concerne les préparations dérivées des corpus de savoirs caractérisés comme "systèmes indiens de médecine », avant tout la médecine indo-persane Unani et la médecine ayurvédique. La question de l'intégration des médecines traditionnelles dans les systèmes de santé des grands pays asiatiques est un enjeu de politiques publiques et une ressource potentielle pour l'accès aux soins depuis les années 1970. À l'époque, tant en Chine qu'en Inde, il s'agit avant tout d'un enjeu professionnel défini en termes de formation des praticiens et de pratiques cliniques. La modernisation est alors un problème d'intégration passant par la définition de curriculum, l'ouverture de facultés délivrant des diplômes, l'évaluation des thérapies, la création d'hôpitaux et de services (Banerjee 2009, Scheid 2002).

\section{Les marchés de la médecine traditionnelle}

Dans ce contexte, l'usage des plantes médicinales et la production des préparations thérapeutiques restait une question médicale et professionnelle, la plupart de ces dernières étant élaborées par les médecins eux-mêmes dans le contexte de la consultation. La principale exception à ce cadrage tenait à l'utilisation par et pour la biomédecine, des principes actifs contenus dans certaines plantes. Certaines, considérées comme particulièrement efficaces, faisaient l'objet de recherche en laboratoires, pour les isoler, les synthétiser et les produire en masse, par l'intermédiaire de la chimie pharmaceutique. C'est ainsi que dans les années 1980 et 1990 la Chine s'engagea, en collaboration avec l'OMS et des firmes pharmaceutiques des Nords, dans un processus de transformation des préparations à base d'Artemisia annua, actives contre le paludisme, en une substance pure, l'artémisinine, élaborée par synthèse partielle à partir d'extraits végétaux, ramenés au statut de matière première. Ces médicaments, recommandés par l'OMS depuis le début des années 2000, constituent aujourd'hui les traitements de première ligne contre la malaria.

La situation a radicalement changé à partir du tournant des années 2000 avec l'émergence d'une dynamique de « pharmaceuticalisation ». Cette dernière fait alors écho aux évolutions de la santé globale, marquée par la place croissante donnée aux médicaments et à leur accès, à la montée en puissance des biotechnologies, à la mondialisation des dispositifs de

\footnotetext{
${ }^{15}$ BTS Report 2016. Pharma Intelligence, UK january 2017.
} 
propriété intellectuelle sur les ressources biologiques, à la croissance des marchés mondiaux du médicament et à la délocalisation partielle de leur production.

Intégrer les médecines traditionnelles d'Asie est ainsi devenu synonyme de promotion de leur industrialisation dans une perspective de création de nouveaux marchés, nationaux et internationaux. Cette évolution, soutenue à la fois par des firmes parfois anciennes et opérant à petite échelle, par les États concernés et par l'OMS, a été particulièrement significative dans le cas de l'Inde (Banerjee 2009, Bode 2008, Pordié and Gaudilliere 2014). À partir des années 2000, Ayush, le département spécialisé du Ministère de la santé, a ainsi recentré ses activités sur la valorisation des mélanges thérapeutiques à base de plantes, soutenant leur inventaire, la mise en culture des végétaux ressources, la standardisation des protocoles et le développement des exportations. Même si ce nouveau marché internationalisé des médicaments traditionnels ne représente qu'une fraction limitée des exportations pharmaceutiques indiennes $(5 \%$ en 2013), le changement d'échelle des opérations des producteurs indiens ainsi que les évolutions de leurs pratiques et celle du modèle économique correspondant ont été spectaculaires. Les grandes firmes du secteur comme Dabur ou Himalaya commercialisent dans toute l'Inde, et dans plusieurs dizaines d'autres pays, des produits dont la présentation est en tous points similaire à celles des comprimés de la biomédecine.

Il faut ainsi parler non seulement d'industrialisation mais aussi de l'émergence d'une forme originale de capitalisme pharmaceutique, largement soutenu par l'État, et qui, comme l'industrie du générique, se pose en alternative aux pratiques d'innovation et de valorisation caractéristiques des grandes entreprises des Nords.

\section{La Traditional Knowledge Digital Library : une alternative aux brevets globalisés}

Les questions de propriété intellectuelle jouent un rôle essentiel dans la stratégie de réinvention des formulations ayurvédiques. Ces dernières sont vues comme des ressources collectives qu'il s'agit de protéger de l'appropriation privée mais également comme des innovations qu'il peut s'agir de breveter. Le statut de la Traditional Knowledge Digital Library, créée par le gouvernement indien en 2000, pour s'opposer aux dépôts de brevets internationaux sur les préparations thérapeutiques ayant pour origine les savoirs médicaux traditionnels de l'Inde, illustre parfaitement cette opposition et le travail de frontières qu'elle implique (Gaudilliere 2014).

La TKDL est en charge d'un travail de traduction des textes décrivant les préparations traditionnelles, pour produire des fiches remettant en ordre ces savoirs par le biais de catégories mimant la structure des brevets. Ces fiches peuvent ainsi servir de ressources dans les controverses sur la propriété intellectuelle. Depuis la signature d'un accord en 2009 entre la TKDL et l'Office Européen des brevets, des dizaines de demandes de brevets ont été annulées pour défaut d'inventivité, sur la base des informations fournies par la base. Pour les autorités indiennes, les formulations classiques sont en effet des ressources nationales à protéger contre la «biopiraterie» occidentale mais dont il s'agit également de favoriser l'usage. Cette protection n'est donc pas incompatible avec l'existence des brevets. De fait, la position défendue par l'Inde dans les négociations internationales sur la protection des savoirs traditionnels n'est pas d'inventer des dispositifs nouveaux et spécifiques mais d'amender les dispositifs mondialisés de la propriété intellectuelle. Parallèlement, au niveau national, l'office indien des brevets a attribué, depuis dix ans, des centaines de brevets, pour la plupart sans validité internationale, sur des reformulations de préparations ayurvédiques ou unanis. Dans cette perspective, la TKDL est, par les informations standardisées digitalisées qu'elle produit, 
un outil de protection mais aussi une ressource à exploiter pour favoriser l'invention par reformulation.

\section{Reformuler et produire des médicaments alternatifs pour des maladies globales}

Le capitalisme «Ayurvédique » repose également sur une stratégie particulière de construction des marchés. L'innovation par reformulation mobilise des savoirs hétérogènes pour produire des réponses alternatives aux problèmes de santé présents dans les Suds et les Nords, comme les pathologies chroniques. Cette dialectique entre tradition et invention est particulièrement visible dans l'activité des firmes les plus importantes, comme la compagnie Himalaya basée à Bangalore.

La firme vend des produits de nature industrielle et mobilise des formes de marketing « scientifique » assez classiques, avec des visiteurs auprès des praticiens. La reformulation reste toutefois une activité distincte de la pharmacie biomédicalisée car l'innovation porte sur des préparations complexes de plantes, des formulations «poly-herbales ». La préparation Menosan proposée comme traitement de la ménopause illustre bien ces pratiques

En dépit du statut pathologique contesté de la ménopause et de son absence du corpus Ayurvédique, Himalaya a développé cette préparation "traditionnelle » pour répondre aux besoins croissants en Inde et ailleurs tout en positionnant ses produits comme réponse à la crise des hormones stéroïdes des années 2000. Dès sa mise sur le marché en 2002, Ménosan est présentée comme une forme naturelle de traitement, basée sur les préparations ayurvédiques. Son invention a reposé sur des allers et retours entre les textes classiques et les laboratoires de la firme pour identifier les formulations ciblant la santé des femmes à cet âge de leur vie et les symptômes de type bouffées de chaleur et troubles de l'humeur. La reformulation (au sens strict de nouvelle combinaison) a consisté, selon une perspective proche du screening, à tester de nombreuses combinaisons nouvelles inspirées de ces formulations de référence pour caractériser leurs effets cliniques.

Construire le marché de Ménosan en alternative à celui des hormones stéroïdes s'est toutefois révélé difficile. Quinze ans après son introduction, le produit est loin d'être un blockbuster. Alors qu'en Europe et aux États-Unis les ventes de stéroïdes ont chuté de 30 à 50 $\%$ depuis 2000, le Menosan se heurte aux régulations. Il n'est pas reconnu comme médicament par la FDA ou l'EMA.

\section{Répliques au Nord : la déstabilisation des systèmes de santé.}

Alors que l'on pensait la division entre les Nords et les Suds bien établie, l'essor des capitalismes de la copie et de la reformulation interroge cette dichotomie entre innovation et production au Nords, pauvreté et aide à l'accès aux Suds. Les ébranlements du grand partage sanitaire résultant des restructurations du capitalisme pharmaceutique ne s'arrêtent toutefois pas à cela. Ils concernent aussi la visibilité croissante des problèmes de pénuries et les difficultés d'accès dans les Nords, comme le révèlent les crises des prix qui se succèdent depuis le début des années 2010.

Des systèmes de santé au nord fragilisés 
Au cours des années 2000, l'épanouissement du capitalisme pharmaceutique des Nords, fondé sur un régime monopolistique alliant biotechnologies, propriété intellectuelle renforcée et financiarisation, est mis à mal. L'arrivée à expiration de nombreux brevets est mal compensée du fait d'une crise de la « productivité pharmaceutique », qui voit la probabilité d'une molécule candidate d'arriver sur le marché décroitre fortement (Pamolli, 2011). La crise financière de 2008 et la volonté des Etats de l'OCDE de contrôler les dépenses liées au médicament, notamment via le développement des génériques, aurait pu fragiliser encore davantage les industriels. Mais, les arbitrages des Etats en faveur d'une protection des marchés du médicament innovant, ont permis aux grands laboratoires de développer de nouvelles stratégies de construction des prix et de valorisation (Abecassis et Coutinet, 2017) qui ont fragilisé les systèmes de santé.

Le capitalisme financiarisé des biotechnologies génère aujourd'hui une inflation du prix des acquisitions de nouvelles molécules par les firmes, qui entraine celle des prix des médicaments. Comme le soulignait une évaluation financière de Gilead en 2015, le modèle du rachat décrit plus haut - fondé sur une R\&D interne plus basse que les autres firmes et sur l'acquisition d'innovation sur le marché des actifs - ne peut fonctionner qu'en parasite sur d'autres formes d'investissements : "Il deviendra difficile de se procurer de tels produits en interne ou en externe (en raison des prix élevés des actifs) ${ }^{16}$. Cette augmentation substantielle des prix des innovations thérapeutiques fait l'objet depuis le début des années 2010 d'une mobilisation visant à la constituer en problème public. En 2014, des ONG ont initié des actions médiatiques et de plaidoyer visant à alerter les organismes de régulation nationaux et européens.

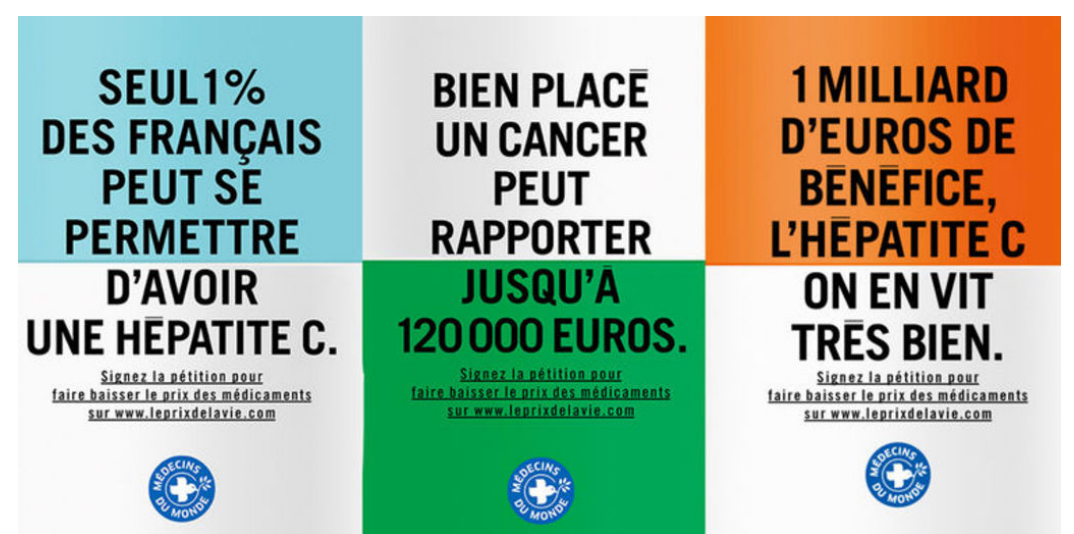

Campagne de Médecins du Monde (2016)

En 2015, dans un rapport remis à la ministre de la Santé, Dominique Polton avertit : «Le défi auquel sont confrontés les systèmes de santé est que, si ces avancées sont bénéfiques pour les patients, les produits concernés atteignent des prix exorbitants qui n'apparaissent plus soutenables » (Polton, 2015, p. 84). Le rapport du CESE ou encore, aux Etats-Unis, celui du Sénat sur le Sovaldi, pointe le problème. Le domaine du cancer est particulièrement identifié ${ }^{17}$. Peter Bach, du Memorial Sloan Kettering, décrit la disproportion entre prix et effets thérapeutiques pour les médicaments anticancéreux mis sur le marché depuis dix ans (David et al., 2015). Pour ces acteurs politiques et bureaucratiques, le "défi » de ce premier quart de siècle pour les systèmes de santé est de tenir trois objectifs : garantir l'accès au soin, promouvoir l'innovation, tenir les dépenses publiques (Brissaud et Juven, 2020).

\footnotetext{
${ }^{16}$ Company note on Gilead Sciences, Jefferies, September 8, 2015, 58 p.

${ }^{17}$ Jean-Paul Vernant, «L'appel de 110 cancérologues contre le coût des traitements », Le Figaro, 14 mars 2016.
} 
La cohabitation de plus en plus difficile entre des systèmes de couverture maladie assurée par la collectivisation des dépenses de santé (quel qu'en soit le mécanisme) et des capitalismes pharmaceutiques financiarisés interroge les modes de régulation des dépenses et la formation des prix des innovations thérapeutiques. Refusant de mobiliser des outils juridiques contraignant comme la licence d'office, les pays du Nord misent depuis vingt ans sur une négociation de prix fondée sur l'évaluation médico-économique de l'efficience des produits. Mais cette architecture est affaiblie dès lors que de certains États régulent les prix a posteriori, en cherchant à limiter les impacts budgétaires (Benoît et Nouguez, 2018) et que de nombreux autres peinent à mobiliser le calcul médico-économique comme outil de négociation. La mise sur le marché d'un médicament, lorsque sa qualité thérapeutique et son coût global sont estimés importants ${ }^{18}$, passe par un processus de justification de son efficience fondée sur des dossiers fournis par les laboratoires aux autorités publiques. Ces dossiers reposent sur des modélisations économiques de type coût-efficacité, mettant en balance la qualité du produit et son coût global. Or ces évaluations sont sujettes à de multiples désaccords (Juven, 2019). Sont mis en question les critères pertinents pour statuer sur le coût global d'un produit, sur son efficacité relative à un comparateur ou encore sur la durée durant laquelle le produit doit être évalué. À titre d'exemple, lorsque le Kymriah, thérapie génique contre le cancer, a été mise sur le marché, Novartis a réclamé un prix de $475000 \$$ par patient aux États-Unis. Le laboratoire a toutefois proposé que le traitement ne soit facturé que pour les patients chez qui une amélioration est détectée au bout d'un mois. L'enjeu devient alors de savoir ce qui doit être considéré comme une amélioration et sur la pertinence de choisir une durée d'un mois (Juven et Bourgain, 2017).

Le Royaume-Uni, connu pour avoir instauré un mécanisme de seuil d'efficience permettant au payeur public de refuser la mise sur le marché d'un traitement considéré comme excessivement cher, pourrait sembler constituer un contre-exemple. Jusqu'en 2011, un seuil d'efficience existait par exemple pour les anti-cancéreux, qui empêchait tout remboursement pour un médicament dont le coût-efficacité excédait la valeur plafond (Devlin et Parkin, 2004). Mais, en créant un fonds public visant à rembourser les médicaments non efficients, le Cancer Drugs Fund, le Royaume-Uni a de facto renoncé à cette politique pour les anticancéreux. De nombreuses analyses, dont celle de la Cour des comptes britannique (National Audit Office, 2015) présentent ce fond comme un gouffre financier ne résolvant pas le problème du financement des anticancéreux (Maynard et Bloor, 2011) et ayant eu pour principal objectif de rendre possible la victoire de David Cameron aux élections de 2010.

Les enjeux de propriété intellectuelle ne sont pas absents de cette situation. Alors que les ONG et les associations de patients tentent d'investir le terrain de l'expertise médicoéconomique, leurs dernières mobilisations ont porté sur la nécessité (en écho au capitalisme de la copie) de rendre rapidement possible la conversion générique d'innovations hors de prix. L'un des cas les plus récents et emblématiques est celui du Truvada, un traitement dans le VIH. Le Truvada est le fait d'un acteur industriel que nous avons déjà mentionné et dont la valeur en bourse est dépendante de la capacité à acquérir des droits de propriété intellectuelle, à les conserver et à les exploiter au maximum dans les pays du nord : le laboratoire Gilead. Alors que de nombreux acteurs dans le domaine de la lutte contre le sida s'attendaient à ce que le Truvada tombe dans le domaine public en 2017, Gilead a déposé en France une demande de certificat complémentaire. Cette procédure, si elle aboutit, permet à l'industriel de bénéficier d'une extension de brevet de cinq ans, et de retarder d'autant l'arrivée sur le marché des génériques.

\footnotetext{
${ }^{18}$ En France par exemple, les médicaments dont le coût sera supérieur à 20 millions d'euros par an pour l'assurance maladie doivent faire l'objet d'une évaluation d'efficience.
} 
En juillet 2017, alors que le brevet est supposé expirer, un génériqueur, Mylan, annonce lancer son générique. Gilead décide alors de poursuivre Mylan en justice et demande au Tribunal de grande instance de Paris l'interdiction provisoire du générique. L'ordonnance du référé rendue le 5 septembre 2017 est on ne peut plus claire : «Statuant publiquement, par remise au greffe, par ordonnance contradictoire et en premier ressort : déboutons les sociétés Gilead de l'ensemble de leurs demandes. Condamnons les sociétés Gilead à payer à la société Mylan la somme de 100000 euros sur le fondement de l'article $700 \mathrm{du}$ code de procédure civile $»^{19}$. Cette décision a été confirmée en 2018 par la Cour de Justice de l'Union Européenne ${ }^{20}$.

\section{Conclusion générale}

Notre analyse des conditions de félicité des capitalismes pharmaceutiques dans les Nords comme dans les Suds permet de saisir la pluralité des agencements par lesquels les industries de santé opèrent voire qu'elles contribuent à structurer en influençant la production des normes et des règles nationales et internationales en matière de science mais aussi de finance et de propriété intellectuelle. Le développement d'un capitalisme centré sur les biotechnologies et s'inscrivant dans les mécanismes des marchés financiers (avec la forte implication des capital-risqueurs, le développement de l'intangibilité du capital scientifique) a constitué pour les Nords, la voie principale d'épanouissement de ce secteur. A contrario, ces modalités d'expansion (biotechnologie et financiarisation) n'ont pu être dupliquées telles quelles dans les Suds ce qui a conduit à l'émergence de capitalismes alternatifs, valorisant la copie ou la reformulation. Toutefois, ces dernières années, des sociétés de biotechnologie chinoises figurent parmi les levées de fonds les plus importantes du venture capital.

Une analyse croisée des conditions d'épanouissement et des épreuves économiques et politiques de l'innovation en santé, englobant les processus de structuration des marchés à la fois dans les Nords et dans les Suds, montre donc qu'il ne saurait être question d'un capitalisme sanitaire mais bien d'une pluralité d'organisations capitalistiques visant à articuler certains objectifs de santé publique (l'accès à des traitements « innovants » ou traditionnels) à des politiques économiques. Cette analyse croisée montre qu'il est possible, en prenant pour point de comparaison le degré de financiarisation, la place de la propriété intellectuelle et l'importance des biotechnologies, d'étudier les conditions de félicité des industries en santé. Ces conditions de félicité, poussées à l'extrême, peuvent menacer la poursuite du régime d'accumulation, se retourner contre les États les ayant promues et devenir des facteurs de crise.

Le modèle développé dans les Nords à partir des années 1970 a vu lui ses vertus (accès rapide à l'innovation, dynamisme financier du secteur des biotechnologies) se retourner contre lui au point de fragiliser l'accès à des traitements de pointe. La montée des tensions sur les prix des médicaments innovants, illustrées par les controverses sur le rationnement du Sovaldi aux États-Unis et en Europe, ou encore par l'intervention des organisations humanitaires et des associations de patients pour faire invalider des brevets en Europe, et non plus seulement en Inde ou au Brésil, interrogent les compromis passés entre firmes pharmaceutiques et payeurs publics ou sociaux depuis la mise en place des couvertures santé dans les années 1940., L'emprise des firmes sur les payeurs est plus forte que jamais, dès lors qu'elles sont parvenues à imposer des formulations de prix fondées sur les gains de valeur thérapeutique des innovations comparés aux traitements existants, sans considération des coûts en capital de la R\&D, sans

\footnotetext{
${ }^{19}$ TGIP, RG 17/57112, 5 septembre 2017.

${ }^{20} \mathrm{http}$ ://www.coalitionplus.org/prix-du-medicament-generique-de-fin-pour-le-monopole-de-gilead-sur-letruvada/?utm source=dlvr.it\&utm medium=twitter
} 
parler des coûts de production et de distribution, et qu'elles réussissent donc à capturer une large part des économies de dépenses de santé permises par ces innovations.

L'irruption du capitalisme de la copie à partir des années 1970, particulièrement en Inde et en Chine, a créé un espace de contestation du capitalisme propriétaire des Nords, qui a en retour motivé la signature d'accords globaux et bilatéraux sur la propriété intellectuelle, imposés par les États Unis et les grands laboratoires dans les années 1990 et 2000, pour limiter ces espaces de libre copie. Simultanément, le capitalisme propriétaire recherche des économies de coûts en sous-traitant la production des principes actifs dans les Suds, au risque d'introduire une nouvelle dépendance et des ruptures d'approvisionnement au nord comme vient de le révéler la crise de la Covid-19. Les capitalismes de la copie se heurtent aussi à des limitations de leurs débouchés, lorsque les couvertures universelles ne permettent pas de rendre les médicaments qu'ils produisent accessibles aux populations les plus pauvres, dans leurs pays ou dans les pays où ils exportent (Afrique).

Un autre déplacement se joue enfin sur le terrain de l'innovation et de sa pluralité. Si la géographie de l'innovation et la répartition de la valeur est encore fortement polarisée vers les Nords (l'Inde est bien le $3^{\text {ème }}$ producteur en volume mais seulement le $9^{\text {ème }}$ en valeur), la croissance accélérée des dépenses d'innovation et des brevets pharmaceutiques en Chine, le couplage, en particulier en Inde, entre médecine traditionnelle et industrialisation, le cas échéant associé à l'extension d'une couverture santé, pourraient signaler un basculement plus profond que celui de la copie dans la mesure où il touche au modèle épistémique qui a nourri le capitalisme propriétaire.

\section{Références}

Abecassis P., Coutinet N., «Politique d'austérité et politique du médicament en France et au Royaume-Uni : Une analyse de leurs répercussions sur le modèle de production pharmaceutique », La Revue de l'Ires, vol. 1, n 91-92, p. 111-140.

Abecassis P., Coutinet N., Économie du médicament, Paris : La Découverte, 2018

Banerjee M. (2009), Power, Knowledge, Medicine. Ayurvedic Pharmaceuticals at Gome and in the World, Hyderabad : Orient Blackswan.

Benoît C., Nouguez E. (2018), « Gouverner (par) les prix. La fixation des prix des médicaments remboursés en France », Revue Française de Sociologie, vol. 58, n 3, p. 399-424.

Boyer R. (2015), Économie politique des capitalismes, Paris : La Découverte.

Brissaud C., Juven P-A. (2020), « Les ruses de la raison budgétaire. L'expertise de l'OCDE sur le prix du medicament ", Actes de la recherche en sciences sociales, $n^{\circ} 234$, pp. 34-49.

Budish E., Roin B., Williams H. (2015), « Do Firms Underinvest in Long-Term Research? Evidence fron Cancer Clinical Trials », American Economic Review, vol. 105, n 7 , p. 2044-2085.

Callon M. (2017), L'emprise des marchés. Comprendre leur fonctionnement pour pouvoir les changer, Paris : La Découverte.

Cassier M 2021, Value regimes and pricing in the pharmaceutical industry: financial capital inflation (hepatitis C) versus innovation and production capital savings for malaria medicines. BioSocieties, Published: 13 January 2021, p 1-19.

Cassier M., Correa M. (2009), « Éloge de la copie : le reverse-engineering des antirétroviraux contre le VIH/ sida dans les laboratoires pharmaceutiques brésiliens », Sciences Sociales et Santé, vol 27, n 3, p. 77-103.

Cassier M., Gaudillière JP. (2000), « Recherche, médecine et marché : la génétique du cancer du sein », Sciences Sociales et Santé, vol 18, n², p 29-51. 
Chauduhri S. (2005), The WTO and India's Pharmaceuticals Industry: Patent Protection, TRIPS, and Developing Countries, Oxford University Press, New Delhi.

Chauveau S. (1999), L'invention pharmaceutique, Paris : la Découverte.

Coriat B., Orsi F. (2002), « Establishing a new intellectual property regime in the United States. Origins, contents and problems », Research Policy, vol. 31, p. 1497-1507.

Cockburn I. (2003), "What a brave new industry that has such patents in it!", Advances in genetics, vol. 50, p. 385-398.

Devlin N., Parkin D. (2004), « Does NICE Have a Cost-Effectiveness Threshold and What Other Factors Influence its Decision? A Binary Choice Analysis », Health Economics, vol. 13, p. 437-452.

Gaudillière J-P. (2014), « An Indian path to biocapital? The Traditional Knowledge Digital Library (TKDL), drug patents and the reformulation regime of contemporary Ayurveda », East Asian Science Technology and Society, vol. 8, p. 391-415.

Gaudillière J-P. (2015), «Une manière industrielle de savoir », in Bonneuil C., Pestre D. (dir.) Histoire des sciences et des savoirs 3 - Le siècle des technosciences, Paris : Seuil, p. 85-105.

Gaudillière J-P. (à paraître), «Innovation and its others: Pharmaceutical Development, Global Capitalism and the Dialectics of Value », Biosocieties.

Goodman J., Walsh S. (2001), The Story of Taxol: Nature and Politics in the Pursuit of an AntiCancer Drug, Cambridge : Cambridge University Press.

Greene J.A., Riggs K.R. (2015) "Why is there no Generic Insulin ? Historical Origines of a modern problem, The New England Journal of Medicine, p 1171-1175.

Howard D., Bach P., Berndt E., Conti R. (2015), " Pricing in the Market for Anticancer Drugs », The Journal of Economic Perpesctives, vol. 29, $\mathrm{n}^{\circ} 1$, p. 139-162.

Joly P-B. (2010), "On the economics of techno-scientific promises », in Callon M., Débordements, mélanges offerts à Michel Callon, Paris : Presses des Mines, p. 203-222.

Jullien B., Smith A. (2012), «Le gouvernement d'une industrie. Vers une économie politique institutionnaliste renouvelée », Gouvernement et action publique, vol. 1, n ${ }^{\circ}$, p. 103-123.

Juven P-A. (2019), « La valeur des médicaments », in Castel P., Juven P-A., Vézian A. (dir.), Les politiques de lutte contre le cancer en France, Rennes : Presses de l'EHESP, p. 181200.

Juven P-A., Bourgain C., "Que valent vraiment les thérapies géniques », The Conversation France, 2017.

Koijen R., Philipson T., Uhlig H. (2016), « Financial Health Economics », Econometrica, vol. $84, \mathrm{n}^{\circ} 1$, p. $195-242$

Lanjouwe J. (1997), The Introduction of pharmaceutical Products Patents in India: « Heartless Exploitation of the poor and suffering? ", Yale : Yale University Press.

Lerner J. (1994), "Venture Capitalists and the Decision to Go Public », Journal of Financial Economics, vol. 35, p. 293-316.

Liebenau J. (1987), Medical Science and Medical Industry: The Formation of the American Pharmaceutical Industry London: Macmillan.

Malki, Elli, Intellectual Property and the Valuation of Biotechnology Companies: Gen-Dex Versus Dow Jones. Available at SSRN:

https://ssrn.com/abstract=43181 or http://dx.doi.org/10.2139/ssrn.43181

Maynard A., Bloor K. (2015), « Regulation of the Pharmaceutical Industry: Promoting Health or Protecting Wealth? », Journal of the Royal Society of Medicine, vol. 108, $\mathrm{n}^{\circ} 6, \mathrm{p}$. 220-222.

Montalban M. (2007), Financiarisation, dynamique des firmes et modèles productifs : une analyse institutionnaliste, Thèse de doctorat, Université Bordeaux IV. 
Mowery, D. and Ziedonis, A. (2000) « Numbers, quality and entry: How has the Bayh- Dole Act affected US university patenting and licensing? » in Jaffe A., Lerner J., Stern, S. (dir.) Innovation and the Economy 1, Cambridge : MIT Press, p.187-220.

National Audit Office (2015), Investigation into the Cancer Drugs Fund.

Pammolli, F., Magazzini, L. and Riccaboni, M. (2011) « The productivity crisis in pharmaceutical R\&D » Nature Reviews Drug Discovery, 10: 428-438.

Polton D., « Rapport des réformes sur les modalités d'évaluation des médicaments », 2015.

Pordié L. \& J-P Gaudillière, «The reformulation strategy in drug discovery : Polyherbals and property rights in the Ayurvedic industry », East Asian Science Technology and Society, 8 (2014) : 57-79.

Quirke V. (2008), Collaborations in the Pharmaceutical Industry. Changing relationships in Britain and France 1935-1965, New York, London, Routledge.

Salbu S. (1993), " Aids and drug pricing. In search of a policy », Washington University Law Review, vol 71, n 3 .

Scheid V. (2002), Chinese Medicine in Contemporary China. Plurality and Synthesis. Durham : Duke University Press.

Scherer F-M., Watal J. (2001), « Post-Trips Options for Access to Medicines in Developing countries », $97 \mathrm{p}$.

Sunder Rajan K. (2017), Pharmocracy. Value, Politics and Knowledge in Global Biomedicine, Durham : Duke University Press.

Swann J-P. (1988), American Scientists and the Pharmaceutical Industry. Cooperative Research in Twentieth-Century America, Baltimore : Johns Hopkins University Press.

Veras J. (2014), Making Tenofovir Accessible in Brazilian Public Health System: Patent

Conflicts and Generic Production, Developing World Bioethics, Aug;14(2):92-100.

Walsh V., Le Roux M. (2004), « Contingency in innovation and the role of national systems: taxol and taxotère in the USA and France », Research Policy, vol. 33 p. 1307-1327. 